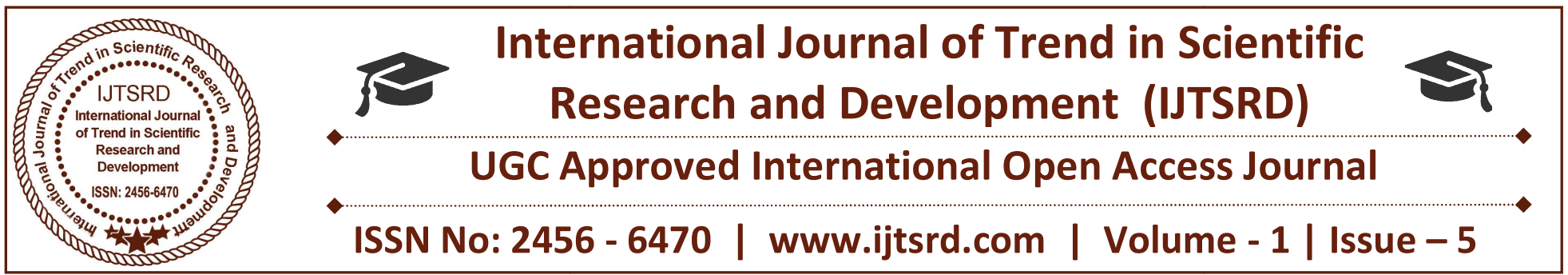

\title{
Fixed Points in Continuous Non Decreasing Functions
}

\author{
Dr. M. RamanaReddy \\ Associate Professor of Mathematics \\ Sreenidhi Institute of Science and Technology, Hyderabad, India
}

\begin{abstract}
The purpose of this paper is to establish a common fixed point theorem is established in G-complete fuzzy metric spaces in the sense of George and Veeramani besides discussing some related problems.
\end{abstract}

Keywords-Continuous t-norm, Fuzzy metric space, G-Cauchy sequence, Housdroff and first countable

\section{INTRODUCTION}

Gregori and Sapena [1] introduced the notion of fuzzy contractive mapping and proved fixed point theorems in varied classes of complete fuzzy metric spaces in the senses of George and Veeramani [2], Kramosil and Michalek [3] and Grabiec's [4]. Soon after, Mihet [5] proposed a fuzzy fixed point theorem for (weak) Banach contraction in M-complete fuzzy metric spaces. In this continuation, Mihet [6,7] further enriched the fixed point theory for various contraction mappings in fuzzy metric spaces besides introducing variants of some new contraction mappings such as: Edelstein fuzzy contractive mappings, fuzzy $\psi$-contraction of $(\epsilon, \lambda)$ type etc. In the same spirit, Qiu et al. [8,9] also obtained some common fixed point theorems for fuzzy mappings under suitable conditions. In 2010 Pacurar and Rus in [10], introduced the concept of cyclic $\phi$-contraction and utilize the same to prove a fixed point theorem for cyclic $\phi$-contraction in the natural setting of complete metric spaces besides investigating several related problems in respect of fixed points.

In this paper the notion of cyclic weak $\phi-$-contraction is introduced in a fuzzy metric space. Furthermore, fixed point theorem is established in G-complete fuzzy metric spaces in the sense of George and Veeramani besides discussing some related problems.

\section{PRELIMINARIES}

Definition -1 . 1 A binary operation $\star:[0,1] \times[0,1] \rightarrow[0,1]$ is continuous $t-$ norm if $\star$ is satisfying the following conditions:

1.1 (i) $\star$ is commutative and associative.

1.1 (ii) $\star$ is continuous.

1.1 (iii) $\mathrm{a} \star 1=$ a for all a $\in[0,1]$.

1.1 (iv) $\mathrm{a} \star \mathrm{b} \leq \mathrm{c} \star \mathrm{d}$ whenever $\mathrm{a} \leq \mathrm{c}$ and $\mathrm{b} \leq \mathrm{d}$,

For $a, b, c, d \in[0,1]$.

Definition - 1.2 A triplet $(X, M, \star)$ is said to be a fuzzy metric space if $X$ is an arbitrary set, $\star$ is a continuous $\mathrm{t}-$ norm and $\mathrm{M}$ is a fuzzy set on $\mathrm{X}^{2} \times(0, \infty)$ satisfying the following condition for all $\mathrm{x}, \mathrm{y}, \mathrm{z}, \mathrm{s}, \mathrm{t}>0$,

$1.2(\mathrm{FM}-1) \mathrm{M}(\mathrm{x}, \mathrm{y}, \mathrm{t})>0$

$1.2(F M-2) M(x, y, t)=1$ if and only if $x=y$.

$1.2(F M-3) M(x, y, t)=M(y, x, t)$ 
$1.2(\mathrm{FM}-4) \mathrm{M}(\mathrm{x}, \mathrm{y}, \mathrm{t}) \star \mathrm{M}(\mathrm{y}, \mathrm{z}, \mathrm{s}) \leq \mathrm{M}(\mathrm{x}, \mathrm{z}, \mathrm{t}+\mathrm{s})$

$1.2(\mathrm{FM}-5) \mathrm{M}(\mathrm{x}, \mathrm{y}, \bullet):(0, \infty) \rightarrow(0,1]$ is continuous.

Then $M$ is called a fuzzy metric on $X$. The function $M(x, y, t)$ denote the degree of nearness between $x$ and $y$ with respect to $t$.

Example 1.3 Let $(X, d)$ be a metric space. Define $a * b=\min \{a, b\}$ and

$$
M(x, y, t)=\frac{t}{t+d(x, y)}
$$

For all $\mathrm{x}, \mathrm{y} \in \mathrm{X}$ and all $\mathrm{t}>0$. Then $(\mathrm{X}, \mathrm{M}, \star)$ is a Fuzzy metric space.

It is called the Fuzzy metric space induced by $d$.

We note that, $M(x, y, t)$ can be realized as the measure of nearness between $x$ and $y$ with respect to $t$. It is known that $M(x, y, \cdot)$ is non decreasing for all $x, y \in X$. Let $M(x, y, \star)$ be a fuzzy metric space for $t>0$, the open ball

$$
B(x, r, t)=\{y \in X: M(x, y, t)>1-r\} .
$$

Now, the collection $\{B(x, r, t): x \in X, 0<r<1, t>0\}$ is a neighborhood system for a topology $\tau$ on $X$ induced by the fuzzy metric M. This topology is Housdroff and first countable.

Definition 1.5 A sequence $\left\{x_{n}\right\}$ in a fuzzy metric space $(X, M, \star)$ is said to be a converges to $x$ iff for each $\varepsilon>0$ and each $t>0, n_{0} \in N$ such that $M\left(x_{n}, x, t\right)>1-\varepsilon$ for all $n \geq n_{0}$.

Definition 1.6 A sequence $\left\{x_{n}\right\}$ in a fuzzy metric space $(X, M, \star)$ is said to be a G- Cauchy sequence converges to $x$ iff for each $\varepsilon>0$ and each $t>0, n_{0} \in N$ such that $M\left(x_{m}, x_{n}, t\right)>1-$ $\varepsilon$ for all $m, n \geq n_{0}$.

\section{MAIN RESULTS}

Theorem - 2.1 Let $(X, M, \star)$ be a fuzzy metric spaces, $A_{1}, A_{2}, \ldots, A_{m}$ be closed subsets of $X$ and $Y=$ $\cup_{i=1}^{m} A_{i}$ be $G-$ complete. Suppose that $\phi:[0, \infty) \rightarrow[0, \infty)$ is a continuous non decreasing function with $\phi(r)>0$ for each $r \in(0,+\infty)$ and $\phi(0)=0$. If $f: Y \rightarrow Y$ satisfying,

$$
\begin{aligned}
\left(\frac{1}{M^{2}(f x, f y, t)}-1\right) \leq\left(\frac{1}{M^{2}(x, f x, t) \star M^{2}(y, f y, t)}-1\right) \\
\quad-\phi\left(\frac{1}{M^{2}(x, f x, t) \star M^{2}(y, f y, t)}-1\right)
\end{aligned}
$$

then $f$ has a unique fixed point $\mathrm{z} \in \cap_{\mathrm{i}=1}^{\mathrm{m}} \mathrm{A}_{\mathrm{i}}$.

Proof : Let $x_{0} \in Y=U_{i=1}^{m} A_{i}$ and set $x_{n}=f x_{n-1}(n \geq 1)$. Clearly, we get $M\left(x_{n}, x_{n+1}, t\right)=M\left(f x_{n-1}, f x_{n}, t\right)$ for any $t>0$. Beside for any $n \geq 0$, there exists $i_{n} \in\{1,2, \ldots, m\}$ such that $x_{n} \in A_{i_{n}}$ and $x_{n+1} \in A_{i_{n}+1}$. Then by $4.2 .4(\mathrm{i})$, (for $\mathrm{t}>0$ ) we have

$$
\begin{aligned}
\left(\frac{1}{\mathrm{M}^{2}\left(\mathrm{x}_{\mathrm{n}}, \mathrm{x}_{\mathrm{n}+1}, \mathrm{t}\right)}-1\right)= & \left(\frac{1}{\mathrm{M}^{2}\left(\mathrm{fx}_{\mathrm{n}-1}, \mathrm{f} \mathrm{x}_{\mathrm{n}}, \mathrm{t}\right)}-1\right) \\
\leq & \left(\frac{1}{\mathrm{M}^{2}\left(\mathrm{x}_{\mathrm{n}-1}, \mathrm{f} \mathrm{x}_{\mathrm{n}-1}, \mathrm{t}\right) \star \mathrm{M}^{2}\left(\mathrm{x}_{\mathrm{n}}, \mathrm{fx}, \mathrm{t}, \mathrm{t}\right)}-1\right) \\
& \quad-\phi\left(\frac{1}{\mathrm{M}^{2}\left(\mathrm{x}_{\mathrm{n}-1}, \mathrm{fx}_{\mathrm{n}-1}, \mathrm{t}\right) \star \mathrm{M}^{2}\left(\mathrm{x}_{\mathrm{n}}, \mathrm{f \textrm {x } _ { \mathrm { n } } , \mathrm { t } )}\right.}-1\right)
\end{aligned}
$$




$$
\leq\left(\frac{1}{M^{2}\left(x_{n-1}, x_{n}, t\right) \star M^{2}\left(x_{n}, x_{n+1}, t\right)}-1\right)
$$

Which implies that

$$
M^{2}\left(x_{n}, x_{n+1}, t\right) \geq M^{2}\left(x_{n-1}, x_{n}, t\right) \star M^{2}\left(x_{n}, x_{n+1}, t\right)
$$

and hence

$$
M\left(x_{n}, x_{n+1}, t\right) \geq M\left(x_{n-1}, x_{n}, t\right) \star M\left(x_{n}, x_{n+1}, t\right)
$$

For all $n \geq 1$ and so $\left\{M\left(x_{n-1}, x_{n}, t\right)\right\}$ is non decreasing sequence of positive real numbers in $(0,1]$.

Let $S(t)=\lim _{n \rightarrow+\infty} M^{2}\left(x_{n-1}, x_{n}, t\right)$. Now we show that $S(t)=1$ for all $t>0$. If not, there exists some $t>0$ such that $\mathrm{S}(\mathrm{t})<1$. Then, on making $\mathrm{n} \rightarrow+\infty$ in 4.2.4(ii), we obtain

$$
\left(\frac{1}{s(t)}-1\right) \leq\left(\frac{1}{s(t)}-1\right)-\phi\left(\frac{1}{s(t)}-1\right)
$$

Which is a contradiction. Therefore $\mathrm{M}^{2}\left(\mathrm{x}_{\mathrm{n}}, \mathrm{x}_{\mathrm{n}+1}, t\right) \rightarrow 1$ as $\mathrm{n} \rightarrow+\infty$. Now for each positive integer $\mathrm{p}$ we have

$$
\begin{array}{r}
M\left(x_{n}, x_{n+p}, t\right) \geq M\left(x_{n}, x_{n+1}, \frac{t}{p}\right) \star M\left(x_{n+1}, x_{n+2}, \frac{t}{p}\right) \\
\star \ldots \ldots \star M\left(x_{n+p-1}, x_{n+p}, \frac{t}{p}\right)
\end{array}
$$

It follows that $\lim _{\mathrm{n} \rightarrow+\infty} \mathrm{M}\left(\mathrm{x}_{\mathrm{n}-1}, \mathrm{x}_{\mathrm{n}}, \mathrm{t}\right) \geq 1 \star 1 \star \ldots \ldots 1=1$

So that $\left\{x_{n}\right\}$ is a G-Cauchy sequence. As $Y$ is G-complete, then there exists $y \in Y$ such that $\lim _{n \rightarrow+\infty} x_{n}=y$. It follows that the iterative sequence $\left\{x_{n}\right\}$ has a infinite numbers of terms in $A_{i}$ for each $i=1,2, \ldots \ldots m$. Since $Y$ is $\mathrm{G}$-complete, for each $A_{i}, i=1,2, \ldots \ldots m$ one can exists a subsequence of $\left\{x_{n}\right\}$ that converges to $y$. By virtue of the fact that each $A_{i}, i=1,2, \ldots, m$ is closed, we conclude that $y \in \cap_{i=1}^{m} A_{i} \neq \phi$. Obviously, $\cap_{i=1}^{m} A_{i}$ is closed and $G$ - complete. Now we consider the restriction of $f$ on $\cap_{i=1}^{m} A_{i}$, i.e. $f \mid \cap_{i=1}^{m} A_{i}: \cap_{i=1}^{m} A_{i} \rightarrow \cap_{i=1}^{m} A_{i}$ thus $f \mid \cap_{i=1}^{m} A_{i}$ has a unique fixed point in $\cap_{i=1}^{m} A_{i}$, say $z$, which is obtained by iteration form the starting point $\mathrm{x}_{0} \in \mathrm{Y}$. To this end, we have show that $\mathrm{x}_{\mathrm{n}} \rightarrow \mathrm{z}$ as $\mathrm{n} \rightarrow \infty$, then, by 4.2.1(i), we have

$$
\left(\frac{1}{\mathrm{M}^{2}\left(\mathrm{x}_{\mathrm{n}}, \mathrm{z}, \mathrm{t}\right)}-1\right) \leq\left(\frac{1}{\mathrm{M}^{2}\left(\mathrm{x}_{\mathrm{n}-1}, \mathrm{x}_{\mathrm{n}-2, \mathrm{t})}\right.}-1\right)-\phi\left(\frac{1}{\mathrm{M}^{2}(\mathrm{fz}, \mathrm{z}, \mathrm{t})}-1\right)
$$

Now letting $\mathrm{n} \rightarrow+\infty$, we get

$\left(\frac{1}{\mathrm{M}^{2}(\mathrm{y}, \mathrm{z}, \mathrm{t})}-1\right) \leq\left(\frac{1}{\mathrm{M}^{2}(\mathrm{y}, \mathrm{y}, \mathrm{t})}-1\right)-\phi\left(\frac{1}{\mathrm{M}^{2}(\mathrm{z}, \mathrm{z}, \mathrm{t})}-1\right)$

Which is contradiction if $\mathrm{M}(\mathrm{y}, \mathrm{z}, \mathrm{t})<1$, and so, we conclude that $\mathrm{z}=\mathrm{y}$. Obviously $\mathrm{z}$ is the unique fixed point of $\mathrm{f}$.

Theorem - 2.2 Let $(X, M, \star)$ be a fuzzy metric spaces, $A_{1}, A_{2}, \ldots, A_{m}$ be closed subsets of $X$ and $Y=$ $\cup_{i=1}^{m} A_{i}$ be $G$ - complete. Suppose that $\phi:[0, \infty) \rightarrow[0, \infty)$ is a continuous non decreasing function with $\phi(\mathrm{r})>0$ for each $\mathrm{r} \in(0,+\infty)$ and $\phi(0)=0$. If $\mathrm{f}: \mathrm{Y} \rightarrow \mathrm{Y}$ satisfying, 


$$
\begin{aligned}
\left(\frac{1}{M^{2}(f x, f y, t)}-1\right) \leq\left(\frac{1}{M^{2}(x, f x, t) \star M^{2}(y, f y, t)}-1\right) & \\
& -\phi\left(\frac{1}{M^{2}(x, f x, t) \star M^{2}(y, f y, t)}-1\right)
\end{aligned}
$$

and there exists a sequence $\left\{y_{n}\right\}$ in $Y$ such that $M\left(y_{n}, f y_{n}, t\right) \rightarrow 1$ as $n \rightarrow+\infty$ for any $t>0$, then $y_{n} \rightarrow z$ as $n \rightarrow+\infty$, provides that the fuzzy metric $M$ is triangular, and $z$ is the unique fixed point of $f$ in $\cap_{i=1}^{m} A_{i}$.

Proof: By Theorem - 4.2.4 we have that $\mathrm{z} \in \mathrm{\cap}_{\mathrm{i}=1}^{\mathrm{m}} \mathrm{A}_{\mathrm{i}}$ is the unique fixed point of $\mathrm{f}$. Now, from the triangular inequality of $\mathrm{M}$ and 4.2.2(i), we have

$$
\begin{array}{r}
\left(\frac{1}{M^{2}\left(y_{n}, \mathrm{z}, t\right)}-1\right) \leq\left(\frac{1}{M^{2}\left(y_{n}, f y_{n}, t\right)}-1\right)+\left(\frac{1}{M^{2}\left(f y_{n}, f z, t\right)}-1\right) \\
\left(\frac{1}{M^{2}\left(y_{n}, z, t\right)}-1\right) \leq\left(\frac{1}{M^{2}\left(y_{n}, f y_{n}, t\right)}-1\right) \\
+\left(\frac{1}{M^{2}\left(y_{n}, f y_{n}, t\right) \star M^{2}(f z, z, t)}-1\right) \\
-\phi\left(\frac{1}{M^{2}\left(y_{n}, f y_{n}, t\right) \star M^{2}(f z, z, t)}-1\right)
\end{array}
$$

Which is equivalent to

$$
\left(\frac{1}{M^{2}\left(y_{n}, z, t\right)}-1\right) \leq\left(\frac{1}{M^{2}\left(y_{n}, f_{n}, t\right)}-1\right)
$$

From the last inequality we conclude that

$$
\lim _{n \rightarrow+\infty} \phi\left(\frac{1}{M^{2}\left(y_{n}, z, t\right)}-1\right)=0
$$

Since

$$
\lim _{n \rightarrow+\infty}\left(\frac{1}{M^{2}\left(y_{n}, f y_{n}, t\right)}-1\right)=0
$$

then, by the property of $\phi$, we conclude that $\mathrm{M}\left(\mathrm{y}_{\mathrm{n}}, \mathrm{z}, \mathrm{t}\right) \rightarrow 1$, which is equivalent to say that $\mathrm{y}_{\mathrm{n}} \rightarrow \mathrm{z}$ as $\mathrm{n} \rightarrow$ $+\infty$.

Theorem - 2.3 Let $(X, M, \star)$ be a fuzzy metric spaces, $A_{1}, A_{2}, \ldots, A_{m}$ be closed subsets of $X$ and $Y=$ $\cup_{i=1}^{m} A_{i}$ be $G$ - complete. Suppose that $\phi:[0, \infty) \rightarrow[0, \infty)$ is a continuous non decreasing function with $\phi(\mathrm{r})>0$ for each $\mathrm{r} \in(0,+\infty)$ and $\phi(0)=0$. If $\mathrm{f}: \mathrm{Y} \rightarrow \mathrm{Y}$ satisfying,

$$
\begin{aligned}
\left(\frac{1}{M^{2}(f x, f y, t)}-1\right) \leq & \left(\frac{1}{M^{2}(x, f x, t) \star M^{2}(y, f y, t)}-1\right) \\
& -\phi\left(\frac{1}{M^{2}(x, f x, t) \star M^{2}(y, f y, t)}-1\right) 2.3(i)
\end{aligned}
$$

and there exists a sequence $\left\{y_{n}\right\}$ in $Y$ such that $M\left(y_{n+1}, f y_{n}, t\right) \rightarrow 1$ as $n \rightarrow+\infty$ for any $t>0$, then there exists $\mathrm{x} \in \mathrm{Y}$ such that $\mathrm{M}\left(\mathrm{y}_{\mathrm{n}}, \mathrm{f}^{\mathrm{n}} \mathrm{x}, \mathrm{t}\right) \rightarrow 1$ as $\mathrm{n} \rightarrow+\infty$ provides that the fuzzy metric $\mathrm{M}$ is triangular.

Proof : Once again, according to the proof of Theorem -4.2 .4 , we observe that for any initial point $x \in$ $\mathrm{Y}, \mathrm{z} \in \mathrm{\cap}_{\mathrm{i}=1}^{\mathrm{m}} \mathrm{A}_{\mathrm{i}}$ is the unique fixed point of $\mathrm{f}$. Moreover, for any $\mathrm{t}>0,0<\mathrm{M}\left(\mathrm{y}_{\mathrm{n}}, \mathrm{z}, \mathrm{t}\right)<1$ and $0<$ $\mathrm{M}\left(\mathrm{y}_{\mathrm{n}+1}, \mathrm{z}, \mathrm{t}\right)<1$. Set $\mathrm{y}$ as a limit of a convergent sequence $\left\{\mathrm{y}_{\mathrm{n}}\right\}$ in $\mathrm{Y}$. Now, from the triangularity of $\mathrm{M}$ and 4.2.6(i), we have 


$$
\begin{aligned}
&\left(\frac{1}{M^{2}\left(y_{n+1}, z, t\right)}-1\right) \leq\left(\frac{1}{M^{2}\left(y_{n+1}, f y_{n}, t\right)}-1\right)+\left(\frac{1}{M^{2}\left(f y_{n}, f z, t\right)}-1\right) \\
&\left(\frac{1}{M^{2}\left(y_{n}, z, t\right)}-1\right) \leq\left(\frac{1}{M^{2}\left(y_{n}, f y_{n}, t\right)}-1\right) \\
&+\left(\frac{1}{M^{2}\left(y_{n}, f y_{n}, t\right) \star M^{2}(z, f z, t)}-1\right) \\
&-\phi\left(\frac{1}{M^{2}\left(y_{n}, f y_{n}, t\right) \star M^{2}(z, f z, t)}-1\right)
\end{aligned}
$$

Then, on making $\mathrm{n} \rightarrow+\infty$ in the above inequality, we get

$$
\begin{aligned}
\left(\frac{1}{M^{2}(y, z, t)}-1\right) \leq & \left(\frac{1}{M^{2}(y, f y, t) \star M^{2}(z, f z, t)}-1\right) \\
& -\phi\left(\frac{1}{M^{2}(y, f y, t) \star M^{2}(z, f z, t)}-1\right)
\end{aligned}
$$

Clearly 4.2.6(ii) is true if and only if $\phi\left(\frac{1}{\mathrm{M}^{2}(\mathrm{y}, \mathrm{z}, \mathrm{t})}-1\right)=0$. By the property of the function $\phi$, must be $\left(\frac{1}{M^{2}(y, z, t)}-1\right)=0$ and thus $y=z$. Consequently, we have $M\left(y_{n}, f^{n} x, t\right) \rightarrow 1$ as $n \rightarrow+\infty$.

\section{REFERENCES}

1) Gregori V., A. Sapena, On fixed point theorems in fuzzy metric spaces, Fuzzy Sets and Systems 125 (2002) 245-253. http://dx.doi.org/ 10.1016/S01650114(00)00088-9.

2) George A., Veeramani P., On some results in fuzzy metric spaces, Fuzzy Sets and Systems 64 (1994) 395-399. http://dx.doi.org/10.1016/01650114(94)90162-7

3) Kramosil O. and Michalek J., Fuzzy metric and statistical metric spaces, Kybernetica 11 (1975), 336-344.

4) Grabiec M. , Fixed points in fuzzy metric spaces, Fuzzy Sets and Systems 27 (1988) 385-389. http://dx.doi.org/10.1016/0165-0114(88)90064-4.

5) Mihet D., A Banach contraction theorem in fuzzy metric spaces, Fuzzy Sets and Systems 144 (2004) 431-439. http://dx.doi.org/10.1016/ S01650114(03)00305-1.
6) Mihet D., On fuzzy contractive mappings in fuzzy metric spaces, Fuzzy Sets and Systems 158 (2007) 915-921. http://dx.doi.org/10.1016 /j.fss.2006.11.012.

7) Mihet D., A class of contractions in fuzzy metrics spaces, Fuzzy Sets and Systems 161 (2010) 11311137. http://dx.doi.org/10.1016 /j.fss.2009.09.018.

8) Qiu D., Shu L., Supremum metric on the space of fuzzy sets and common fixed point theorems for fuzzy mappings, Inform. Sci. 178 (2008) 35953604. http://dx.doi.org/10.1016/j.ins.2008.05.018.

9) Qiu D., Shu L., Guan J., Common fixed point theorems for fuzzy mappings under $\phi$-contraction condition, Chaos Solitons Fractals 41(2009) 360367.

http://dx.doi.org/10.1016/j.chaos.2008.01.003

10) Pacurar M., Rus I.A., Fixed point theory for $\phi$ contractions, Nonlinear Anal. 72 (2010) 11811187. http://dx.doi.org/10.1016/j.na.2009.08.002. 